\title{
Fuzzy Inference Procedure for Intelligent and Automated Control of Refrigerant Charging
}

\section{The link to the formal publication is via}

https://doi.org/10.1007/s40815-018-0486-3

\author{
Issam W. Damaj · Jean J. Saade · Hala R. Al-Faisal · Hassan B. Diab
}

Received: date / Accepted: date

\begin{abstract}
Fuzzy logic controllers are readily customizable in natural language terms, and can effectively deal with non-linearities and uncertainties in control systems. This paper presents an intelligent and automated fuzzy control procedure for the refrigerant charging of refrigerators. The elements that affect the experimental charging and the optimization of the performance of refrigerators are fuzzified and used in an inference model. The objective is to represent the intelligent behavior of a human tester, and ultimately make the developed model available for the use in an automated data acquisition, monitoring, and decision-making system. The proposed system is capable of determining the needed amount of refrigerant in the shortest possible time. The system automates the refrigerant charging and performance testing of parallel units. The system is built using data acquisition systems from National Instruments and programmed under LabVIEW. The developed fuzzy models, and their testing results, are evaluated according to their compatibility with the
\end{abstract}

Issam W. Damaj

Department of Electrical and Computer Engineering, American University of Kuwait, Salmiya, Kuwait

Tel.: +9651802040

E-mail: idamaj@auk.edu.kw

Jean J. Saade

Department of Electrical and Computer Engineering, American University of Beirut, American University of Beirut, Beirut, Lebanon

Hala R. Al-Faisal

Department of Computer Engineering, Kuwait University, Khaldiya, Kuwait

Hassan B. Diab

Department of Electrical and Computer Engineering, American University of Beirut, Beirut, Lebanon principles that govern the intelligent behavior of human experts when performing the refrigerant charging process. In addition, comparisons of the fuzzy models with classical inference models are presented. The obtained results confirm that the proposed fuzzy controllers outperform traditional crisp controllers and provide major test time and energy savings. The paper includes thorough discussions, analysis, and evaluation.

Keywords Refrigerant charging · Modeling human expertise $\cdot$ Performance $\cdot$ Fuzzy inference $\cdot$ LabVIEW

\section{Introduction}

The manufacturing of refrigerators comprises thorough electromechanical safety and a variety of performance tests. Safety tests are performed per international standards and can include circuit, voltage, insulation, power, current leakage tests, etc. Well designed, reliable and validated performance tests are critical for quality production. Both safety and performance tests can be automatic or semi-automatic, and supported by a variety of equipment, data acquisition (DAQ) systems, and human operators [1,2].

Refrigerator performance tests vary in type and duration. Tests can be long-term with durations between 1 to 3 hours, or short-term with durations between 10 and 45 minutes. Further tests can be applied to samples of production over a duration between 3 and 12 hours. Tests in thermo-regulated rooms can last between 12 and 72 hours. Performance tests include temperature readings from the refrigerator main parts, such as, the compartments and evaporator, besides its ambient. Moreover, the tests include careful control of refrigerant charge quantities to attain the best possible per- 
formance and enable sufficient cooling and avoid various hazards [3].

Due to the important effect of the amount of refrigerant charge on the performance of refrigerating units, the influence of the amount of refrigerant on the functioning of these units has been addressed in [4,5]. Traditionally, the optimization of the amount of charge has been done experimentally and either manually or semiautomatically. However, refrigerant charging is proven to require time and labor and also consumes energy [4, 6. In the literature, soft computing and classical methods to develop, computerize and automate the control and testing of refrigerators have been given a wide attention $7,8,9,10,11,12,13,14$. The aims of the identified studies, however, differ from the objectives of this investigation.

The experimental setting of refrigerant charging optimization can become more appealing if the process is automated, while accounting for the reduction of the time, labor, and energy factors. Fuzzy logic and inference methodology can be employed to emulate the intelligent behavior of human experts in the control process. Fuzzy inference has been essentially developed for the design of humanistic or approximate reasoning systems [15, 16, 17]. Fuzzy control could fit well the humanperformed and/or semi-automatic optimization of the refrigerant charging and testing processes.

In this paper, we investigate the automation of the refrigerant charging and performance testing processes. The aims comprise saving time, labor, and energy while maintaining the processes adequacy. The investigation includes the development of a fuzzy inference, a classical control, and refrigerant charging and testing DAQ systems. The developed systems are implemented under LabVIEW and targets DAQ devices from National Instruments [18, 19]. The proposed system supports parallel analysis, testing, and charging of several refrigerators.

This paper is organized so that Section 2 presents related works. Section 3 discusses the motivation and research objectives. In Section 4 , background information is presented. In Section 5 the expert testing procedure is presented. Sections 6 and 7 present the developed classical and fuzzy solutions. Section 8 presents the system architecture and implementation. Thorough analysis and evaluation are presented in Section 9. Section 10 concludes the paper and sets the ground for future work. The Appendix include a list of acronyms and symbols, that are used in the paper, and their definitions.

\section{Related Work}

Automated and fuzzy operation control, and performance testing, of refrigerators are widely addressed in the literature. The aim of investigations includes optimizing performance, simplifying control procedures, and/or mimicking the behavior of a human operator. Filho et al. in [7] developed an adaptive fuzzy controller for a 5-ton vapor compression system. The developed system achieved high coefficient of performance $(C O P)$ values at relatively low rotation frequencies. Yang et al. in 8 proposed a self-adjusting fuzzy controller to improve the performance of refrigerators and their power efficiency. The developed system supports self-adjusting the fuzzy rules in real-time. Kocyigit in [9] successfully analyzed faulty conditions of a refrigeration system using fuzzy inference system and artificial neural networks. The developed system-fault and sensor-error analysis procedures are based on the thermodynamic properties and refrigeration cycles of the system. You et al. in 10 investigated the optimization of the performance of refrigerators under large-scale load variations using fuzzy control. Significant energy saving is reported at an increased $C O P$. Saving energy and improving the $C O P$ is also investigated by $\mathrm{Li}$ and Fei in 11. The investigation included fuzzy control, for the capacity and superheat, to avoid the complexity of exact dynamic models and take the advantage of mimicking the human operator behavior with simplicity.

A classical step-wise optimization of charge amount is presented in [14] for the Joule-Thomson cooler. The proposed optimization procedure successfully identified the best mixed-charge amounts. The lowest refrigeration temperature of $-139{ }^{\circ} \mathrm{C}$ is obtained after $134 \mathrm{~min}$ utes of running time. The required amount of charge to achieve optimal performance, is commonly known to depend on the evaporator and condenser capacities, outer heat transfer surfaces, the capacity of the capillary tube, the ambient temperature, etc. [5, 20].

Damaj et al. in 6] investigate the refrigerant charging process using fuzzy control within a factory performance testing and quality control experimental setup. The developed fuzzy controller is basic and aims at mimicking the behavior of a human operator. Significant time savings in the testing procedure are reported while maintaining the adequacy of the $C O P$ and the quality of the tested system. The study is limited to a single experiment setup and did not include an evaluation of the system impact on energy consumption or labor. In addition, the setup does not include an automated refrigerant charging system. 


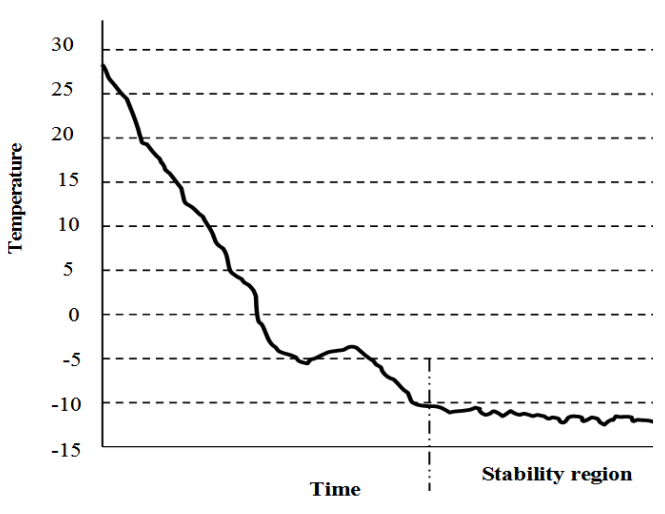

Fig. 1 The change in temperature versus time.

\section{Research Objectives}

The purpose of the refrigerant charging process is to find the amount of refrigerant needed to attain the highest $C O P$ at the best possible temperature. With no doubt, rapidly deciding on the optimal amount with enough assurance is of various advantages. Advantages include saving testing time, labor, and energy. Experimental charging is done through repeated addition of a specific amount of refrigerant, and monitoring the temperature change for each obtained amount as a function of time. The monitored temperature is usually taken as the average internal temperature of the refrigerator cabinet obtained by considering the low and high temperature sections as well as their capacities. Every time an amount of refrigerant is added, the minimum possible attainable temperature should be detected in as small time as possible.

In the targeted refrigeration systems, the regular trend of temperature change with time is decreasing in nature (See an approximation in Figure 1). Accordingly, if the period over which this change is observed, as it starts to become insignificant, is made shorter, then the risk of detecting a temperature larger than the optimum becomes higher. The way to deal with the stated problem is to place appropriate emphasis on the correlation between the length of the observation interval and the extent of the temperature change. The objective is to make the observation interval correspond closely and be fairly sufficient relative to the desired stable temperature range. The aim is to allow for the detection of the lowest or close to the lowest temperature, while not requiring too much time to finish the testing process.

Considering the problem statement and the concise description of its solution procedure, fuzziness arises naturally. For instance, fuzziness is involved in the terms: shorter, insignificant, much larger, fairly sufficient, close to the lowest, and too much time. These terms can be represented by fuzzy sets with suitable membership functions. In addition, fuzzy if-then inference rules can be structured to fit the problem description and provide appropriate solutions [15, 16, 17]. The fuzzy models that are to be devised would have to represent, therefore, how a human expert performs the refrigerant charging process. Inference rules, which can be employed in the fuzzy models, can be of the form shown in Equation 1

- If temperature change is significant and observation time is insufficient, then testing continues.

- If temperature change is insignificant and observation time is fairly sufficient, then testing stops.

The purpose of this paper is to use fuzzy logic and inference methodologies to pave the way for the emergence of an intelligent and automatic control procedure for the process of refrigerant charging. A detailed description and more justification of the use of the fuzzy inference rules and the results they provide along with comments on these results are provided in Sections 4, 6 and 7. Furthermore, since limited inference-based control methods for refrigerant charging of refrigerators have been reported in the literature, non-fuzzy inference models based on crisp sets or intervals are presented in Section 6 for comparison purposes. We should mention at this point that fuzzy and other soft computing techniques were introduced and used in the refrigeration applications $[7,8,9,10,11,12,13$. However, the aims of the reported work in the literature differ from the objectives of the subject addressed in this paper. In this paper, we go beyond the work presented in [6] with focus on achieving better time savings, higher performance, investigating the impact on energy consumption and labor use, and providing a real-time system that is capable of testing parallel refrigerators inside or outside thermos-regulated rooms. The motivations behind this paper are summarized as follows:

- Automating the performance testing of refrigerators is a useful industrial application and a challenging process control procedure. The automation targets a widely-used appliance and enables a broad impact.

- The refrigerant charging process can be time consuming, labor intensive, and consumes high energy in parallel-testing setups. To that end, the opportunity for multiple improvements are present.

- The design of a tight control system requires access to the full state vector of the underlying system; in practice, this is not always feasible [21. The presence of the human factor, in this problem, cannot be modeled or implemented using a classical controller with the usual mathematical formulations at 
a reduced complexity. Mimicking the behavior of a human operator, and exploiting the intrinsic fuzziness of the problem, promise significant reductions in the control system complexity - given the availability of modern rapid-prototyping tools and the exclusion of the human operator.

- Automating the process of monitoring and refrigerant charging improves the reliability of the procedure with the elimination of the chance of human errors. Besides, it increases the safety of the process.

The research objectives are summarized as follows:

- Reduce the time of the refrigerant charging procedure and performance testing of refrigerators, while maintaining the quality of the product.

- Capture the intrinsic fuzziness of the process to provide accurate control at reduced complexity.

- Develop a fuzzy system that captures the decisionmaking process of a human operator.

- Develop a classical control model for the sake of results comparison and system evaluation.

- Develop a testing system using high-end DAQ equipment that support parallel testing of many refrigerators. The system includes sensors, interfaces, hardware, and application software graphical user interface $(G U I)$.

- Present a thorough analysis and evaluation of the proposed solution, and investigate possible improvements in testing time, control performance, labor needs, and power consumption.

\section{Background}

\subsection{Fuzzy Sets and Fuzzy Logic}

The concept of a fuzzy set 22] consists of a generalization of the concept of a classical set. An element in a space over which a fuzzy set is defined can have a partial grade of membership in a fuzzy set instead of being either a member or not a member as in a classical set. Hence, the notion of a characteristic function is generalized to become a membership function mapping the space over which the fuzzy set is defined to the interval $[0,1]$. This makes the membership function represent a smooth rather than an abrupt transition from complete membership to non-membership. Here, a fuzzy set becomes an entity having no sharply defined boundary.

Fuzzy logic emerges from the concept of a fuzzy set to deal with fuzzy or vague linguistic propositions arising in natural languages. A simple fuzzy proposition, therefore, is not necessarily true or false. It can have a degree of truth in the interval $[0,1]$. Some of the membership functions ( $M F s$ ) used to describe a fuzzy

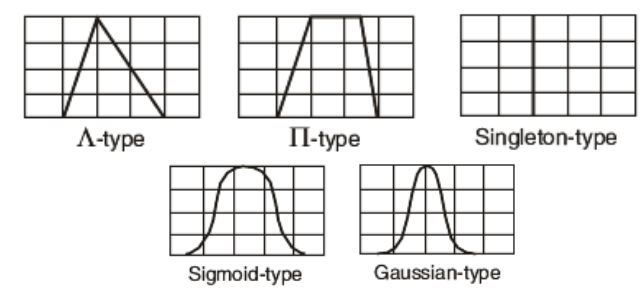

Fig. 2 Different types of membership functions.

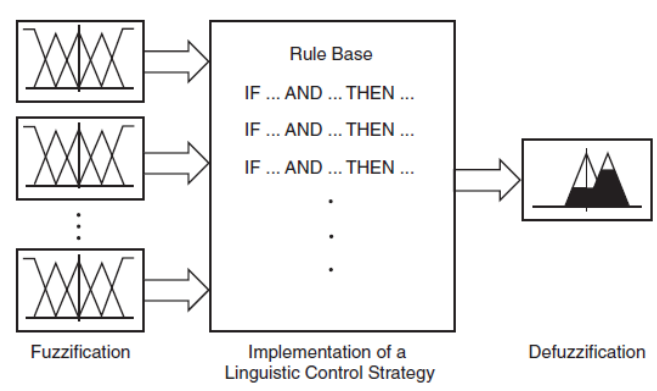

Fig. 3 A fuzzy controller model.

set are shown in Figure 2, 18, 15, 23, 24, 25, 26]. Simple fuzzy propositions can be combined by the logic operators $A N D$ and $O R$, for example, to yield composite propositions. The THEN operator can also be used to construct conditional fuzzy propositions (fuzzy $I F$ THEN rules), which form the backbone of fuzzy inference systems (See Equation 1) or fuzzy controllers.

\subsection{Fuzzy Controllers}

A fuzzy controller is an input-output system formed by a fuzzifier, a defuzzifier and a collection of fuzzy inference rules (See Figure 3). Having fuzzy sets (overlapping ones) assigned over the input and output variables of the fuzzy controller, then the IF-THEN rules provide the necessary connection between these input and output fuzzy sets. For crisp input values, the membership grades in the input fuzzy sets are obtained by fuzzification. The fuzzified grades are then used in the rules setting and by applying the compositional rule of inference $(C R I)$ to obtain a corresponding fuzzy output, which needs to be defuzzified; i.e. converted into a crisp output. Indeed, various defuzzification methods are devised in the literature [26, 27, 28, 29].

Let an N-rule, two-input, one-output fuzzy system be such that the $j^{\text {th }}$ rule, $1 \leq j \leq N$, is expressed in Equation 2.

$R_{j}: \boldsymbol{I F}$ xis $A_{j} \boldsymbol{A N D}$ y is $B_{j}, \boldsymbol{T H E N} z$ is $C_{j}$ 
$A_{j}, B_{j}$ and $C_{j}$ are fuzzy sets assigned over $x, y$ and $z$ which are, respectively, the input and output variables of the system. The $C R I$-obtained fuzzy output, $C_{0}$, and corresponding to the crisp input pair $\left(x_{0}, y_{0}\right)$ is as in Equation 3

$$
\mu_{C_{0}}(z)=\max _{1 \leq j \leq N}\left[\mu_{A j}\left(x_{0}\right) \wedge \mu_{B j}\left(y_{0}\right) \wedge \mu_{C j}(z)\right] .
$$

The minimum operator $(\wedge)$ is used to represent the $A N D$ and THEN operators. The $O R$ operator, that is usually considered between the rules, is represented by maximum (max). If crisp values, $C_{j} \mathrm{~s}$, are assigned over the controller output variable, making the THEN parts of the rules crisp, then a crisp output value corresponding to $\left(x_{0}, y_{0}\right)$ can be obtained directly from within the rules without passing a fuzzy output. The weighted average defuzzification formula, expressed in Equation 4, is one way to obtain this crisp output [17, 29]:

$$
C_{0}=\frac{\sum_{j=1}^{N} \mu_{j} \times c_{j}}{\sum_{j=1}^{N} \mu_{j}}, \text { where } \mu_{j}=\mu_{A j}\left(x_{0}\right) \wedge \mu_{B j}\left(y_{0}\right)
$$

\subsection{Analysis and Design of Fuzzy Control Systems}

The relationship between fuzzy logic and linear system theories motivates numerous investigations on the systematic analysis and design of fuzzy control systems. Accordingly, important investigations related to fuzzy system stability analysis have comprised component failures, reliable control, effects of time delays, chaotic modes, performance degradations, applications, etc. $30,31,32,33,34$.

\section{Expert Testing Procedure}

The usual refrigerant charging procedure is intrinsically fuzzy. Undoubtedly, the observation of temperature change, at various refrigerant amounts, and the time spent to attain this temperature, depend on the level of expertise of the hu-man tester. A conservative tester with less expertise would, for example, extend the observation time as much as possible and maybe beyond what is necessary to complete the process. In such a situation, the test could have been stopped at an earlier time without having a significant effect on the reached temperature. Such an earlier and more adequate stop would mostly be done by a less conservative tester with a higher level of expertise. The experienced tester can correlate in a proficient manner between various ranges (or approximate ranges) of temperature change and various corresponding observation time periods (or approximate periods) to decide whether the test should continue or stop.

In fact, the consideration of approximate ranges of temperature change and lengths of observation time periods can suitably be represented by fuzzy sets and logic. The fuzzy controller can be developed based-on how an expert interprets the curve in Figure 1 to implement the above-mentioned correlation and translate it in the form of decision-making strategies. Now, the main question would therefore be: How should the change in temperature be concretely considered relative to the time that has elapsed to reach the desired point? In other words, how the stoppage or continuation of the testing process should be decided?

It can be argued that the temperature change and observation time variables could be partitioned into ranges. Moreover, the stoppage or continuation of the testing procedure could be made to depend on each combination of two intervals, one for temperature change and the other for observation time. In fact, this constitutes the basis for a plausible classical solution whose main aspects are presented in Section 6 .

A more natural type of a solution and closer to how the testing procedure is usually performed by a human expert can be based on fuzzy logic and inference. Instead of partitioning the problem variables into intervals, the partitioning can be done into fuzzy intervals or equivalently fuzzy sets. Then, pair-wise combinations of these sets are to be structured and corresponding outputs, related to the stoppage (STOP) or continuation $(C O N T)$ of the testing, are to be assigned to give fuzzy inference rules.

\section{Classical Solutions}

In this section, and consistently with what has been presented in Section 5 two classical inference models are provided for the control of the refrigerant charging process. The first solution consists of partitioning the temperature change variable, denoted Dtemp, into two intervals: $0 \leq$ Dtemp $<0.5$, and Dtemp $\geq 0.5$. The observation time variable, denoted Time, is also partitioned into two intervals: $0 \leq$ Time $<30$ minutes and Time $\geq 30$ minutes. The corresponding classical rules are presented in Equation 5

If $0 \leq$ Dtemp $<0.5{ }^{\circ} \mathrm{C}$ and Time $\geq 30 \mathrm{~min}$, then STOP

If $0 \leq D$ temp $<0.5{ }^{\circ} \mathrm{C}$ and $0 \leq \mathrm{Time}<30 \mathrm{~min}$, then CONT

If Dtemp $\geq 0.5{ }^{\circ} \mathrm{C}$ and Time $\geq 30 \mathrm{~min}$, then CONT

If Dtemp $\geq 0.5{ }^{\circ} \mathrm{C}$ and $0 \leq$ Time $<30 \mathrm{~min}$, then CONT 


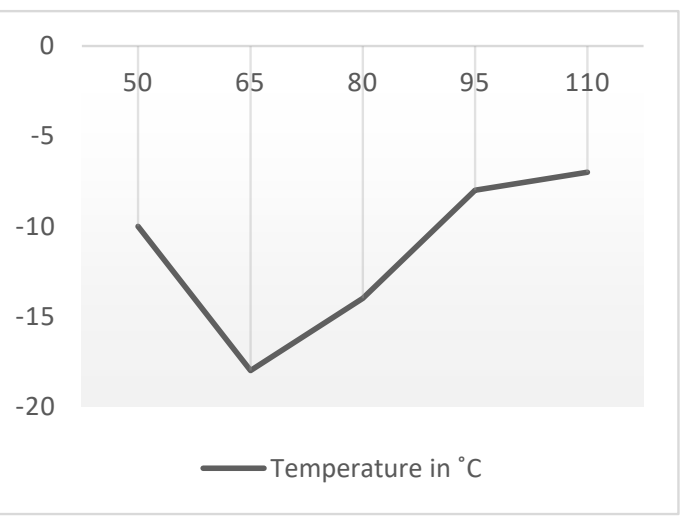

Fig. 4 Refrigerant amount in grams vs. min. temperature.

The implementation of the first classical solution considers the use of a timer that starts recording the time when Dtemp reaches a value less than 0.5. The scanning stops only when Dtemp becomes less than 0.5 ${ }^{\circ} \mathrm{C}$ for an observation time greater than or equal to 30 minutes. A charging situation is assumed here so that the noted observation period is considered as one that leaves no doubt about reaching the stability stage and thus recording the smallest temperature that can be achieved for a specific amount of refrigerant.

More emphasis on the correspondence between the temperature change and observation time, as it relates to various refrigerant-charging situations, will be given in the sequel. When the scanning stops, another amount of refrigerant is added and the process is repeated. In Figure 4, a chart is drawn showing the refrigerant amount versus the minimum temperature. Thus, the amount of refrigerant needed to give the highest possible $C O P$ can be found. But, this is not in as small a time as possible. Since, after reaching a value less than $0.5{ }^{\circ} \mathrm{C}$, Dtemp could even become smaller requiring a stoppage time instant before 30 minutes. Such a possibility needs to be accounted for; this can be done as in a second classical controller.

An improvement to the solution in Equation 5 is to make it softer and hence closer to human experts testing (See Section 5). The improvement can be done by providing additional interval partitioning to the variables. For Dtemp, The intervals are: $\left[0,0.25{ }^{\circ} \mathrm{C}\right),[0.25$ $\left.{ }^{\circ} \mathrm{C}, 0.35{ }^{\circ} \mathrm{C}\right),\left[0.35{ }^{\circ} \mathrm{C}, 0.5^{\circ} \mathrm{C}\right)$ and $\left[0.5{ }^{\circ} \mathrm{C}, \infty\right)$. For Time, the intervals expressed in minutes are: $[0,20),[20,25)$, $[25,30)$ and $[30, \infty)$. Considering all the pair-wise combinations of these intervals and assigning an appropriate stop or continue testing for each combination, while respecting the principles of Section 5 , then 16 rules are result (See Equation 6).

If $0 \leq$ Dtemp $<0.25{ }^{\circ} \mathrm{C}$ and $0 \leq$ Time $<20 \mathrm{~min}$, then CONT If $0 \leq$ Dtemp $<0.25{ }^{\circ} \mathrm{C}$ and $20 \leq$ Time $<25 \mathrm{~min}$, then STOP
If $0 \leq$ Dtemp $<0.25{ }^{\circ} \mathrm{C}$ and $25 \leq$ Time $<30 \mathrm{~min}$, then STOP

If $0 \leq$ Dtemp $<0.25{ }^{\circ} \mathrm{C}$ and Time $\geq 30 \mathrm{~min}$, then STOP

If $0.25 \leq$ Dtemp $<0.35{ }^{\circ} \mathrm{C}$ and $0 \leq$ Time $<20 \mathrm{~min}$, then CONT

If $0.25 \leq$ Dtemp $<0.35{ }^{\circ} \mathrm{C}$ and $20 \leq$ Time $<25 \mathrm{~min}$, then CONT

If $0.25<$ Dtemp $<0.35{ }^{\circ} \mathrm{C}$ and $25<$ Time $<30 \mathrm{~min}$, then STOP

If $0.25 \leq$ Dtemp $<0.35{ }^{\circ} \mathrm{C}$ and Time $\geq 30 \mathrm{~min}$, then STOP

If $0.35 \leq$ Dtemp $<0.5{ }^{\circ} \mathrm{C}$ and $0 \leq$ Time $<20 \mathrm{~min}$, then CONT

If $0.35 \leq$ Dtemp $<0.5{ }^{\circ} \mathrm{C}$ and $20 \leq$ Time $<25 \mathrm{~min}$, then CONT

If $0.35<$ Dtemp $<0.5{ }^{\circ} \mathrm{C}$ and $25 \leq$ Time $<30 \mathrm{~min}$, then CONT

If $0.35<$ Dtemp $<0.5{ }^{\circ} \mathrm{C}$ and Time $>30 \mathrm{~min}$, then $S T O P$

If Dtemp $\geq 0.5{ }^{\circ} \mathrm{C}$ and $0 \leq$ Time $<20 \mathrm{~min}$, then CONT

If Dtemp $\geq 0.5{ }^{\circ} \mathrm{C}$ and $20 \leq$ Time $<25 \mathrm{~min}$, then CONT

If Dtemp $\geq 0.5{ }^{\circ} \mathrm{C}$ and $25 \leq$ Time $<30 \mathrm{~min}$, then CONT

If Dtemp $\geq 0.5{ }^{\circ} \mathrm{C}$ and Time $\geq 30 \mathrm{~min}$, then CONT

Now, considering the 6 rules in Equation 6 with stop testing outputs, then these 6 rules can be combined to become only 3 rules. The remaining 10 rules lead to continuing the testing procedure. Accordingly, Equation 6 is reduced to Equation 7 .

If $0 \leq$ Dtemp $<0.25{ }^{\circ} \mathrm{C}$ and Time $\geq 30 \mathrm{~min}$, then STOP If $0.25<$ Dtemp $<0.35{ }^{\circ} \mathrm{C}$ and Time $\geq 30 \mathrm{~min}$, then STOP If $0.35<$ Dtemp $<0.5{ }^{\circ} \mathrm{C}$ and Time $>30 \mathrm{~min}$, then STOP Otherwise, then CONT

\section{The Fuzzy Inference Model Solution}

The fuzzy control model is formed by fuzzy sets and inference rules of the type given in Equation 11 and thus reflecting the natural fuzziness of the problem. The model used in the testing procedure is depicted in Figure 5 with all the necessary hardware for interfacing and measurements as explained in the sequel. The main fuzzy controller uses the Multiple Input Single Output (MISO) control configuration shown in Figure 6. The controller has the two inputs Dtemp and Time, and an output indicating the required charging action. Here, the $(M F s)$ of fuzzy sets are assigned over and representing a partitioning of these variables. The inference rules consist of all pair-wise combinations of these fuzzy sets, where each combination takes one set from each variable. The controller output is related to the stoppage or continuation of the testing procedure.

The ranges of the controller variables, fuzzy sets, and the inference rules, are assigned based on consultations with experts in the refrigeration field. The consultations also helped improve the contents of Sections 3. 5, and 6, which are also relied upon. Furthermore, some tuning has been done to the fuzzy sets and rules to adjust the model and make it, along with its results, more consistent with the expectations of the experts.

The Dtemp variable has a range between 0 and $5{ }^{\circ} \mathrm{C}$ and 4 fuzzy sets denoted as Very Small, Small, Medium 


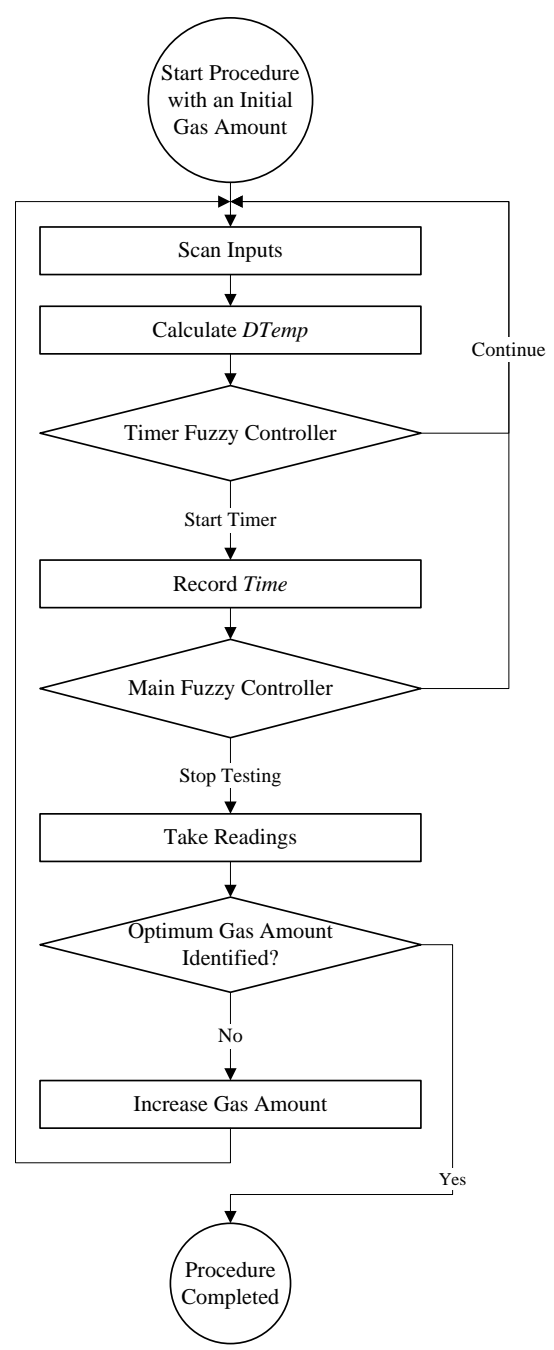

Fig. 5 Refrigerant charging algorithm.

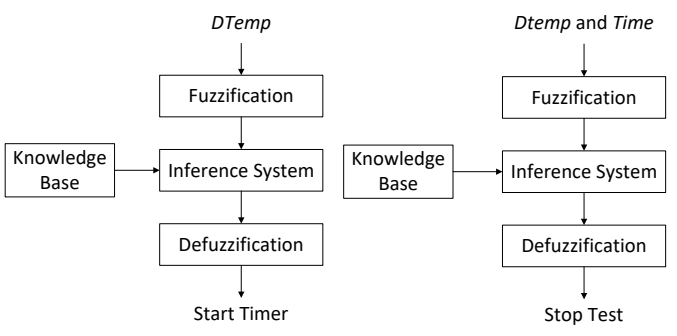

Fig. 6 MISO control configuration of the timer and main fuzzy controllers.

and Large with $M F$ s shown in Figure 7. As they relate to the problem description of Section 3 and the linguistic terms used, the fuzzy sets used here can be interpreted as insignificant, fairly insignificant, significant and highly significant respectively. The Time variable has a range between 0 and 40 minutes and the 5 fuzzy sets Very early, Early, Right, Late, and Very Late with $M F$ s shown in Figure 8, The linguistic interpretation

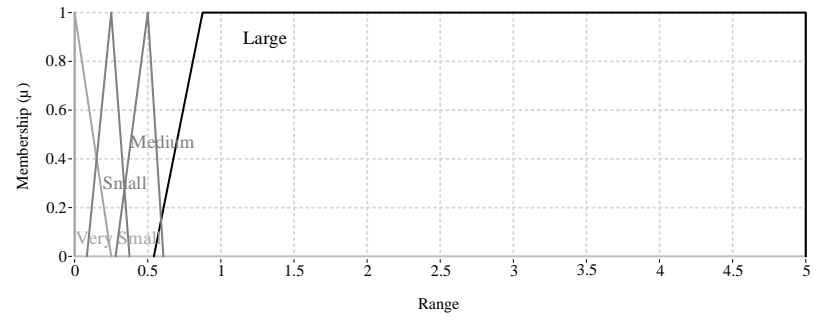

Fig. 7 MFs of Dtemp.

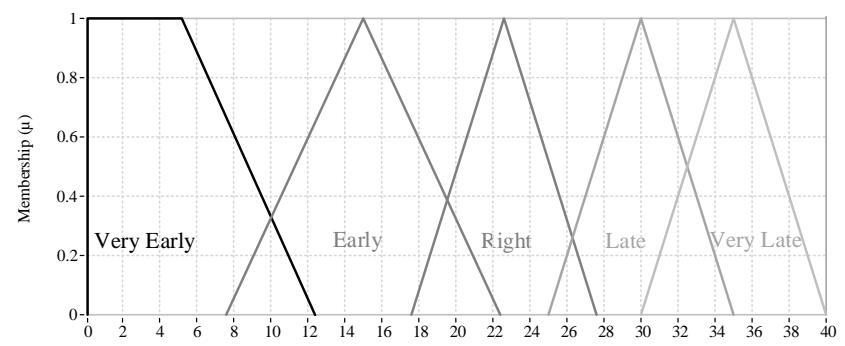

Range

Fig. 8 MFs of Time.

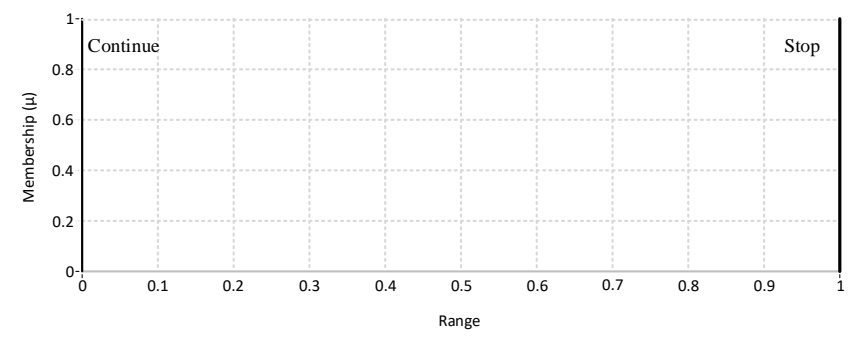

Fig. 9 MFs of the main fuzzy controllers output.

of these fuzzy sets, as they relate to the time required to finish the testing process, can respectively be considered as insufficient, fairly insufficient, sufficient, fairly sufficient and highly sufficient. The output variable of the fuzzy controller, denoted Output, has two singleton terms at 0 and 1 and they are respectively called Stop and Continue (See Figure 9). Based on the above partitioning, the main fuzzy controller uses the 20 inference rules given in Table 10 .

The choice of $M F s$ is of direct impact on the overall performance of the fuzzy system. Trapezoidal membership functions are formed using straightlines; thus, they are simple to capture and implement. Due to their smooth shape, Gaussian MFs can provide appealing controller characteristics as they are non-zero at almost all points [28,35. A close examination of the inference rules structure reveals that the principle that should govern the intelligent behavior of a human expert is respected (See Section 5). In addition, the fuzzy sets MFs are selected so that, when they are used with the rules in Table 10, they would give an approximate 
Table 1 Inference rules of the main controller.

\begin{tabular}{l|ccccc}
\hline DTemp & Very Early & Early & $\underline{\underline{\text { Rime }}}$ & & \\
\hline \hline Very Small & Cont. & Stop & Stop & Stop & Very Late \\
\hline Small & Cont. & Cont. & Stop & Stop & Stop \\
\hline Medium & Cont. & Cont. & Cont. & Stop & Stop \\
\hline Large & Cont. & Cont. & Cont. & Cont. & Cont. \\
\hline
\end{tabular}

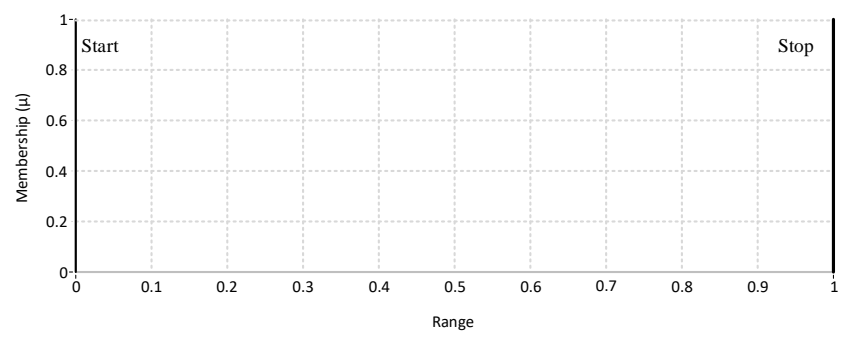

Fig. 10 MFs of the timer fuzzy controllers output.

stoppage time consistent with the expectations of the experts. Here, the opinion of the expert plays the main role in the representation of $M F s$. If the rules in row 1 in Table 10 are considered, then these rules imply that for Dtemp near $0.1^{\circ} \mathrm{C}$ ( Very Small), the stoppage time needs to be near 15 minutes (Early). The rules in the second row imply that for Dtemp near $0.25^{\circ} \mathrm{C}$ (Small), then the stoppage time needs to be near 23 minutes (Right). The third row imply that if Dtemp is close to $0.5{ }^{\circ} \mathrm{C}$ (Medium), then the stoppage time needs to be close to 30 minutes (Late).

In fact, the 15, 23, and 30 minutes mentioned above are reasonable if the timing starts after having Dtemp small enough as determined by the timer fuzzy controller whose rules can be set conveniently by the designer (See Equation 8). Yet, the stoppage time instants can be changed easily and conveniently by tuning the fuzzy sets and rules to accommodate for various charging situations.

The Timer fuzzy controller has two variables; Dtemp and Output. The two variables have the same MFs as those in the main fuzzy controller. However, the output singletons are Stop and Start (See Figure 10). The inference rules of the timer controller are shown in Equation 8

If Dtempis Very Small, then Output is Start

If Dtemp is Small, then Output is Start

If Dtemp is Medium, then Output is Start

If Dtemp is Large, then Output is Stop

A combination triangular and trapezoidal MFs are used for the representation of DTemp and Time as in

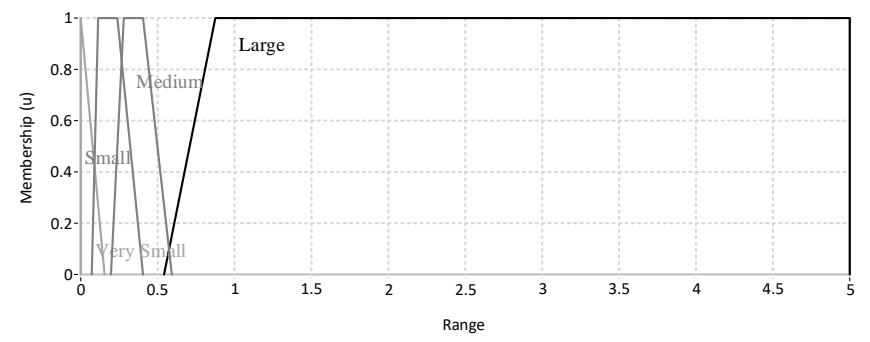

Fig. 11 Trapezoidal MFs of Dtemp.

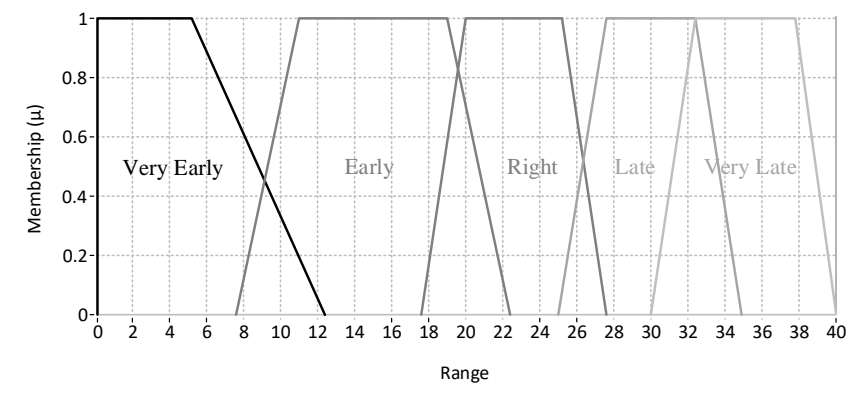

Fig. 12 Trapezoidal MFs of Time.

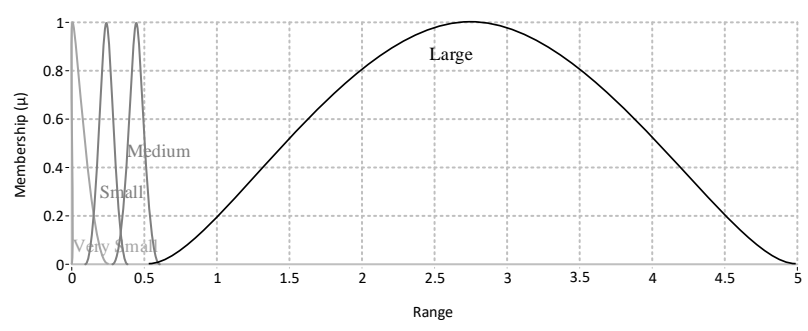

Fig. 13 Gaussian MFs of Dtemp.

Figures 7 and 8 . However, fully-trapezoidal and Gaussianonly alternatives are included in the investigation (See Figures 11, 12, 13, and 14.

\section{System Architecture and Implementation}

The algorithm in Figure 5 is implemented as part of a computerized $D A Q$, monitoring and decision-making system used for refrigerant charging, testing and performance evaluation of refrigerators. The system is implemented under LabVIEW using the available Fuzzy 


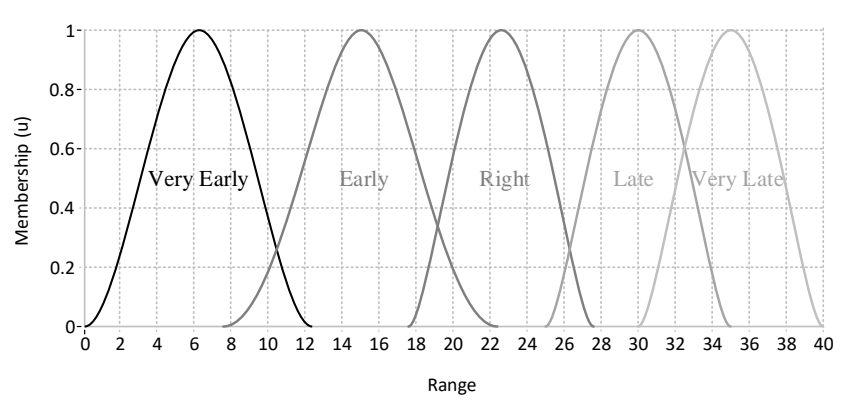

Fig. 14 Gaussian $M F s$ of Time.

Logic Toolkit and contains a complete hardware system for interfacing and measurement. Using the Fuzzy Logic Toolkit functions, the design and tuning of the fuzzy logic controller, as well as the activation of the inference engine for real-time process control, can be easily done.

Two software processes, a scanner and an analyzer, are included in the testing procedure shown in Figure 5. The scanning process acquires the temperature readings from the thermocouples connected to the hardware interfacing cards and controls the reading speed. The analyzer processes the data acquired by the scanner. In addition, the analyzer monitors the changes in temperature reading and records the lowest attained temperature once the main controller decides to stop the testing for a specific refrigerant amount. As the charging is repeated, until the optimum amount is identified, the analyzer draws a chart of refrigerant amount versus temperature as in Figure 4.

The model illustrated in Figure 15 consists of two out of eight fridges being charged in parallel. The NIcDAQ9174 DAQ Chassis is used along with three NI 9211 Thermocouple Modules. Six thermocouples are used for each fridge at various locations to guarantee the accuracy of the process. After acquiring the required data from the thermocouples attached to the three NI 9211 Thermocouple Modules, it gets processed by the developed software to determine Dtemp and accordingly the Time, Start, Continue, and Stop of the timer and the refrigerant charging along with the amount of gas required. The refrigerant charging unit is controlled using normally-closed solenoid valves and NI DAQ C-Series Relay Output Modules.

The main elements of the GUI include the freezer and gas controllers (See Figure 16). The GUI also includes monitors for the temperature change at different parts of the refrigeration system. The temperature monitoring for every part of the system has thermocouple type, module, and, physical channel list selectors. The module list selector is used for choosing the des-

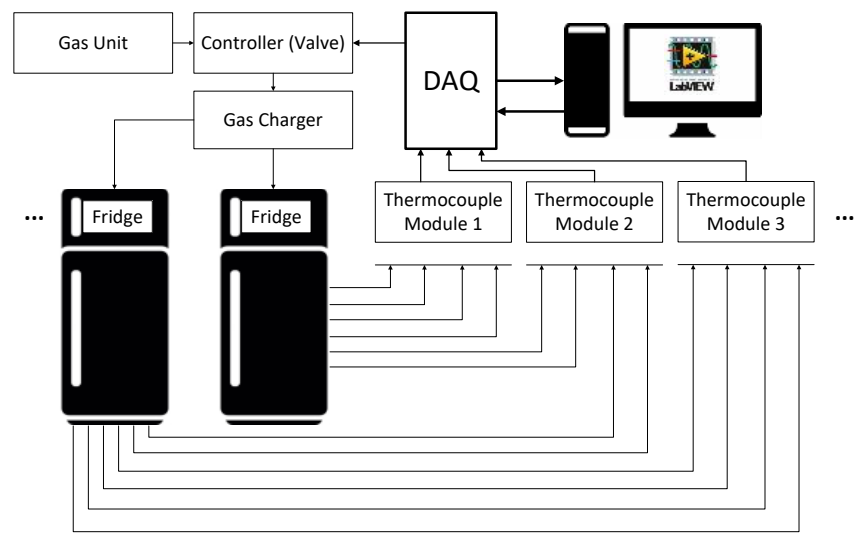

Fig. 15 Gas Charging System Architecture. Two fridges out of eight are shown.

ignated $D A Q$ module as several modules are mounted on each $D A Q$. The physical channel specifies one out of eight possible thermocouples, per $D A Q$, to monitor. Several temperature indicators are added for each stage of monitoring (scanning and analyzing).

The scanning and analyzing using the fuzzy controllers are applied on the freezer part of the refrigerator. The freezer monitoring window includes a state indicator that reflects the status of the procedure while analyzing and can be either in progress or stopped. The freezer window also includes a temperature waveform charts that plots the temperature versus time. Lastly, a timer in minutes is added to the freezers window for noting the time the process is taking.

The gas monitoring window comprises a quantity indicator, and a waveform chart that depicts the freezers minimum temperature at stability versus the gas amount in grams. Moreover, the user inter-face includes waveform charts to monitor the state of the temperature throughout the stages and visually asserting whether stability has been reached or not (See Figure 17). For one unit, six thermo-couples are distributed among the ambient, fridge, freezer, evaporator-in, evaporator-out, and the suction tube $\left(S_{4}\right)$. As the application software supports eight units, the user has the option to hide parts of the GUI window if a smaller number of units are being tested.

\section{Analysis and Evaluation}

\subsection{Results}

Based on the MFs in Figure 7, the rules in Equation 8 . and Equation 5 , the output of the timer fuzzy controller changes per Dtemp values. As the values change between the specified margins, the degree of belonging- 


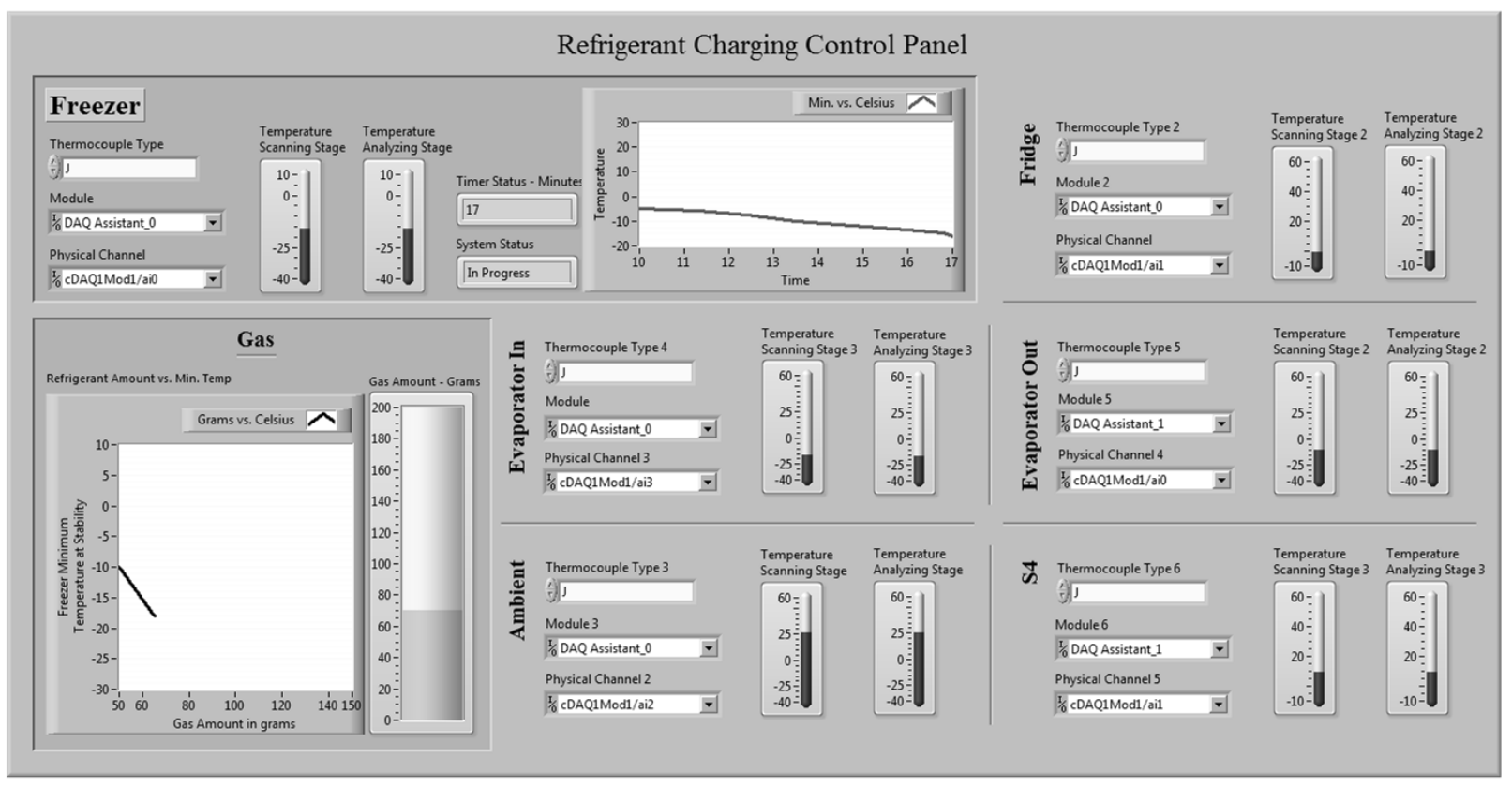

Fig. 16 The refrigerant charging main control panel.

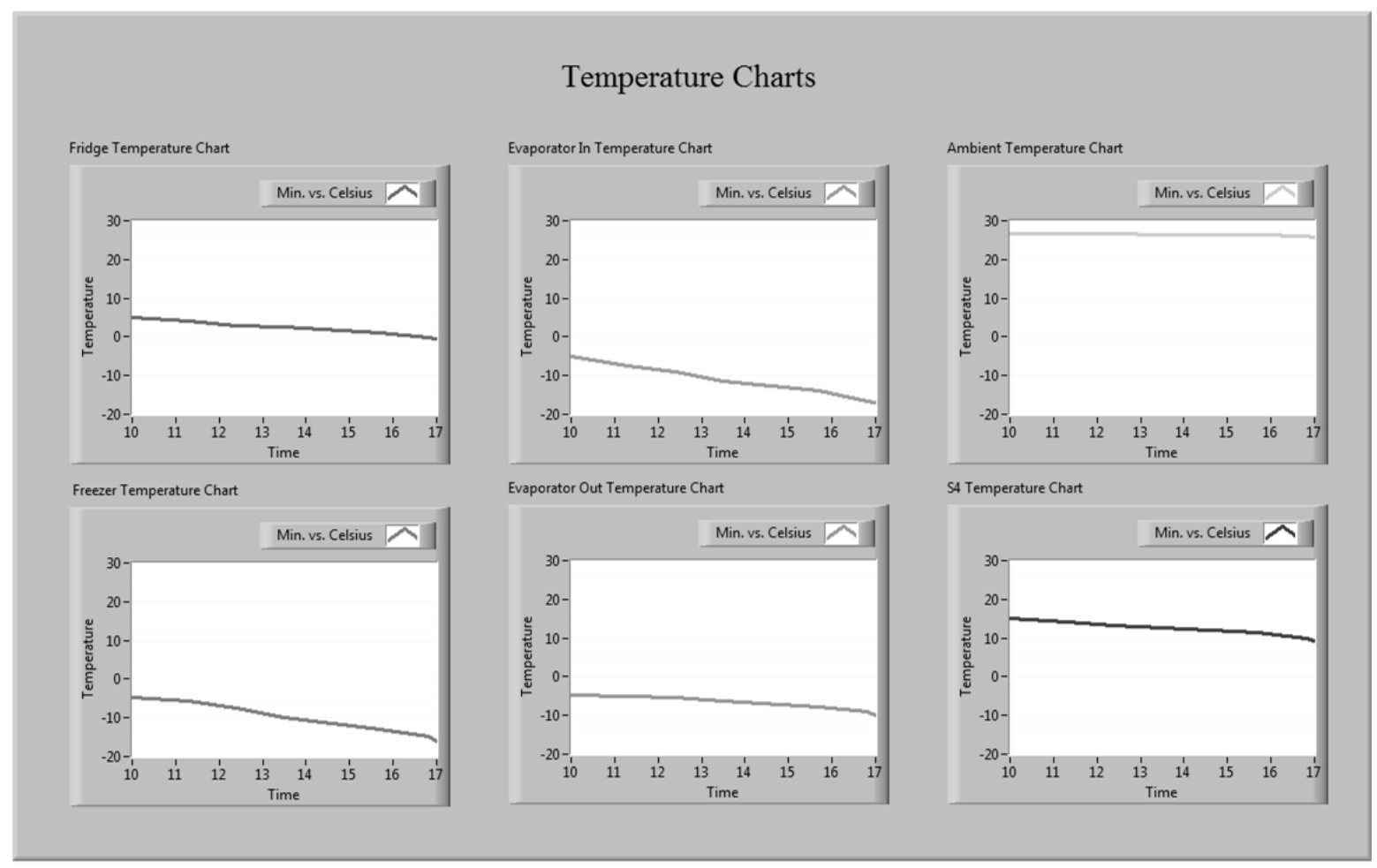

Fig. 17 The refrigerant charging temperature monitoring charts. 


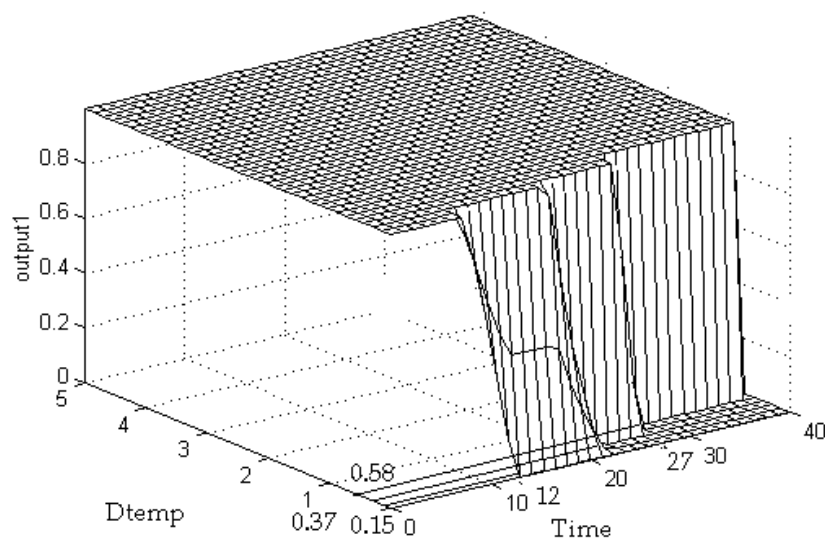

Fig. 18 The output of the main fuzzy controller.

ness to each of the Continue or Stop fuzzy sets changes accordingly. Setting a threshold value somewhere between $0.5{ }^{\circ} \mathrm{C}$ and $0.75{ }^{\circ} \mathrm{C}$ to start the timer shows the acquired ability to modify the start time in accordance with the way by which the human expert would perceive the Dtemp values as indicators for reaching the stability region. If necessary, the range of Dtemp threshold can be modified as well by varying the rules in Equation 8. Thus, the value of Dtemp at which the timer starts can be adjusted in accordance with the human expertise.

According to the MFs in Figure 7 and Figure 8, the rules in Table 10, and Equation 7, Figure 18 shows the main controllers output versus Dtemp. Thus, if Dtemp is in the range $\left[0{ }^{\circ} \mathrm{C}, 0.15{ }^{\circ} \mathrm{C}\right]$, then the controller stops the test in 12 minutes. Now, if Dtemp is between (0.15 ${ }^{\circ} \mathrm{C}, 0.37^{\circ} \mathrm{C}$ ], then the controller stops the testing in 20 minutes. For Dtemp in $\left(0.37^{\circ} \mathrm{C}, 0.58{ }^{\circ} \mathrm{C}\right]$, the controller waits more time to stop the test (27 minutes). Moreover, if the value of Dtemp is between $\left(0.58{ }^{\circ} \mathrm{C}, 5{ }^{\circ} \mathrm{C}\right]$, then the controller keeps the test running. These results are reasonable in terms of their compatibility with the experts expectations as considered while setting the membership functions and rules in Section 7

The plot of the main-controller output versus Dtemp and Time (Figure 18) shows that when Dtemp becomes smaller, then the observation period is also made smaller permitting an earlier stoppage time instant. In addition, the 12, 20 and 27 minutes are not the only time values at which the testing can stop. When the timer starts, Dtemp values could, for example, be in the range $\left[0.37{ }^{\circ} \mathrm{C}, 0.58{ }^{\circ} \mathrm{C}\right]$ leading to an anticipated stoppage time at 27 minutes. But, as the time progresses these values of Dtemp could decrease and become in $\left[0.15^{\circ} \mathrm{C}, 0.37^{\circ} \mathrm{C}\right]$ and then in $\left[0{ }^{\circ} \mathrm{C}, 0.15^{\circ} \mathrm{C}\right]$. When such a decrease happens, the time could have reached any value between 12 and 20 minutes at which the testing stops. Also, the passage of Dtemp values from the first range to the second could occur at any time between 20 and 27 minutes. To that end, the stoppage time of the test can be at any value between 12 and 27 minutes. Hence, the continuity aspect, which can be attributed to human behavior especially in the control area, is obtained here.

To evaluate the performance of the proposed fuzzy control algorithms we develop the following indicators 36 , 37, 38:

1. Test Time $(\boldsymbol{T T})$ : The time, in Minutes (min), taken to complete the test of one unit.

2. Time Saving $(\boldsymbol{T S})$ : The difference between $T T$ s of two controllers.

3. Throughput $(\boldsymbol{T H})$ : The number of tests per unit time in Tests per Hour $(T p H)$ or Tests per a 12-hour work Day $(T p D)$.

4. Performance $(\boldsymbol{P R})$ : The $P R$ of a controller is inversely proportional to its $T T$.

The $P R$ indicator is used to calculate Performance Ratios $\left(P R_{\text {ratio }}\right)$ 38] according to Equations 9 and 10 The $P R$ indicator enables the calculation of the percentage performance improvement of one controller over the other.

$P R_{\text {ratio }}=\frac{P R_{\text {controller }}}{P R_{\text {controller }}}$

where $P R_{\text {ratio }}$ is in $\%$, and

$\frac{P_{\text {controller }_{1}}}{P R_{\text {controller }_{2}}}=\frac{T T_{\text {controller }_{2}}}{T T_{\text {controller }_{1}}}$

5. Energy Consumption $(\boldsymbol{E})$ : in kilo Watts per hour $(k W h)$.

With an aim to experiment test time savings, different membership functions are investigated. Table 2 . presents the implementation results of triangular, trapezoidal and Gaussian MFs. From Table 2, it can be clearly seen that the Fuzzy controllers outperformed the crisp controllers. Nevertheless, minimal performance differences are detected between different types of membership functions for the developed fuzzy controller. The trapezoidal function provides the best performance for the designated Dtemp ranges with a $70.6 \%$ performance improvement based-on the $P R_{\text {ratio }}$ and a maximum $T S$ of $21.2 \mathrm{~min}$, followed by the Gaussian with $60 \%$, then the triangular with $36.6 \%$. The performance difference is due to the different shapes of the MFs which affects the calculation of the degree of membership to a certain fuzzy set. In the presented TT measurements, the valve dynamics can be ignored relevant to the dominant dynamics of the slow temperature process. With a maximum close or open time of 1 Second, and the small amount of gas charge increment, the solenoid valve operation and the refrigerant charging introduce no significant time delays to the overall process 39]. 
Table 2 The measured $T T$ at different values of Dtemp with the $T S$ and $P R_{\text {ratio }}$ of the different controller implementations over the crisp controller of Equation 5

\begin{tabular}{|c|c|c|c|c|c|}
\hline DTemp Range & $\begin{array}{c}\text { Crisp } \\
\text { Equation } 5\end{array}$ & $\begin{array}{c}\text { Crisp } \\
\text { Equation } 7\end{array}$ & $\begin{array}{c}\text { Controllers } \\
\text { Fuzzy } \\
\text { Triangular } \\
\text { MFs }\end{array}$ & $\begin{array}{c}\text { Fuzzy } \\
\text { Trapezoidal } \\
\text { MFs }\end{array}$ & $\begin{array}{c}\text { Fuzzy } \\
\text { Guassian } \\
\text { MFs }\end{array}$ \\
\hline $0.4 \leq$ DTemp $\leq 0.5$ & $30 \mathrm{~min}$ & $30 \mathrm{~min}$ & 27 min & {$[26,27] \min$} & $26.2 \min$ \\
\hline$T S$ & - & $0 \min$ & $3 \min$ & {$[4,3] \min$} & $3.8 \mathrm{~min}$ \\
\hline$\%$ Improvement based-on $P R_{\text {ratio }}$ & - & $0 \%$ & $10 \%$ & {$[13 \%, 10 \%]$} & $12.6 \%$ \\
\hline $0.35 \leq D T e m p \leq 0.4$ & $30 \mathrm{~min}$ & $30 \mathrm{~min}$ & {$[25,27] \min$} & {$[26,27] \min$} & $26.2 \min$ \\
\hline$T S$ & - & $0 \min$ & {$[5,3] \min$} & {$[4,3] \min$} & $3.8 \mathrm{~min}$ \\
\hline$\%$ Improvement based-on $P R_{\text {ratio }}$ & - & $0 \%$ & {$[13 \%, 10 \%]$} & {$[13 \%, 10 \%]$} & $12.6 \%$ \\
\hline $0.25 \leq$ DTemp $\leq 0.3$ & $30 \mathrm{~min}$ & 25 min & 20 min & {$[19.1,20] \min$} & $\overline{19.3 \mathrm{~min}}$ \\
\hline$T S$ & - & $5 \min$ & $10 \mathrm{~min}$ & {$[10.9,10] \min$} & $10.7 \min$ \\
\hline$\%$ Improvement based-on $P R_{\text {ratio }}$ & - & $16.7 \%$ & $33.3 \%$ & {$[33.6 \%, 33.3 \%]$} & $35.6 \%$ \\
\hline $0 \leq D$ Demp $\leq 0.25$ & $30 \mathrm{~min}$ & 20 min & {$[12,20] \min$} & {$[8.8,19.1] \mathrm{min}$} & {$[19.1,19.3] \mathrm{min}$} \\
\hline $\begin{array}{c}T S \\
\% \text { Improvement based-on } P R_{\text {ratio }}\end{array}$ & $\begin{array}{l}- \\
- \\
\end{array}$ & $\begin{array}{c}10 \min \\
33 \% \\
\end{array}$ & $\begin{array}{l}{[18,10] \min } \\
{[60 \%, 33.3 \%]}\end{array}$ & $\begin{array}{l}{[21.2,10.9] \mathrm{min}} \\
{[70.6 \%, 36.3 \%]} \\
\end{array}$ & $\begin{array}{c}{[10.9,10.7] \min } \\
{[36.3 \%, 35.6 \%]} \\
\end{array}$ \\
\hline
\end{tabular}

With no doubt, the time saving leads to significant reduction in energy consumption of the testing process. The energy saving is proportional to the time saving given Equation 11 and can reach a maximum of $70.6 \%$ saving per unit using the fuzzy controller with trapezoidal MFs. We consider that a modern refrigerator unit consumes a total power $(P)$ of $2.5 \mathrm{~kW}$ 40.

$E=P \times t$

Where, $t$ is the time in hours, $E$ in $k W h$, and $P$ in $k W$

Figure 19 shows the number of tests per time that can be run versus time-given a maximum setup time of 15 minutes per test. Here, a test means that the minimum temperature has been detected once a new amount of refrigerant has been added. Usually, to decide on the best gas amount, 4-6 tests are required. The figure considers the worst and best calculated time savings for the crisp and fuzzy controllers. The Figure shows that the crisp controller of Equation 6 performed almost equally to the fuzzy controller with trapezoidal $M F s$ but at its worst-case duration of test. The fuzzy controller with trapezoidal MFs outperformed all other models in its best-case time saving of $70.6 \%$.

Table 3 and Figure 20 show the calculated energy consumption per test versus time. The Table considers the worst and best test times for both the crisp and fuzzy controllers. The results in the Table confirms that the Trapezoidal MFs enable the highest $T H$ of 30 $T p D$. The Figure shows that the energy consumption per test per unit is almost constant over time with a value of $1 \mathrm{kWh}$. The results shown in the table are for the controller with trapezoidal MFs at its best-case test time.

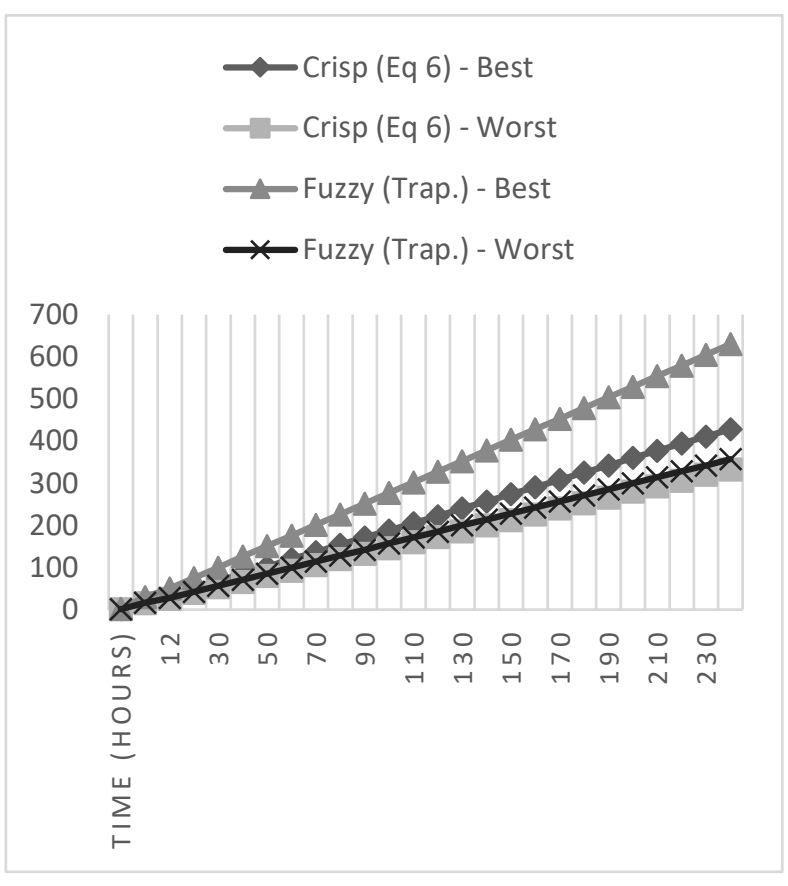

Fig. 19 The number of tests versus time for one unit.

\subsection{General Evaluation}

In addition to the above-mentioned results, provided by the fuzzy models, Table 4 contains comparisons among the fuzzy model of Table 10 and the classical inference model of Equation 7. The main differences between the two models are the complexity of required procedural support to the software tool, and the complexity of the development and tuning of the model. The main complexity is caused by the procedures which need to 
Table 3 Energy consumption per test for the worst and best times for both of crisp and fuzzy controllers. The maximum test setup time is considered 15 minutes, while the power consumption is $2.5 \mathrm{~kW}$. The results shown are for one unit.

\begin{tabular}{l|l|l|l}
\hline Controller & Test Time in minutes & No. of Tests in 12 Hours & E per Test $\mathrm{kWh} /$ test \\
\hline \hline Crisp (Equation 7) - Best & 20 & 20 & 1.5 \\
\hline Crisp (Equation 7 ) - Worst & 30 & 16 & 1.87 \\
\hline Fuzzy (Trapezoidal $M F s$ ) - Best & 8.8 & 30 & 1 \\
\hline Fuzzy (Trapezoidal $M F s$ ) - Worst & 27 & 17 & 1.76 \\
\hline
\end{tabular}

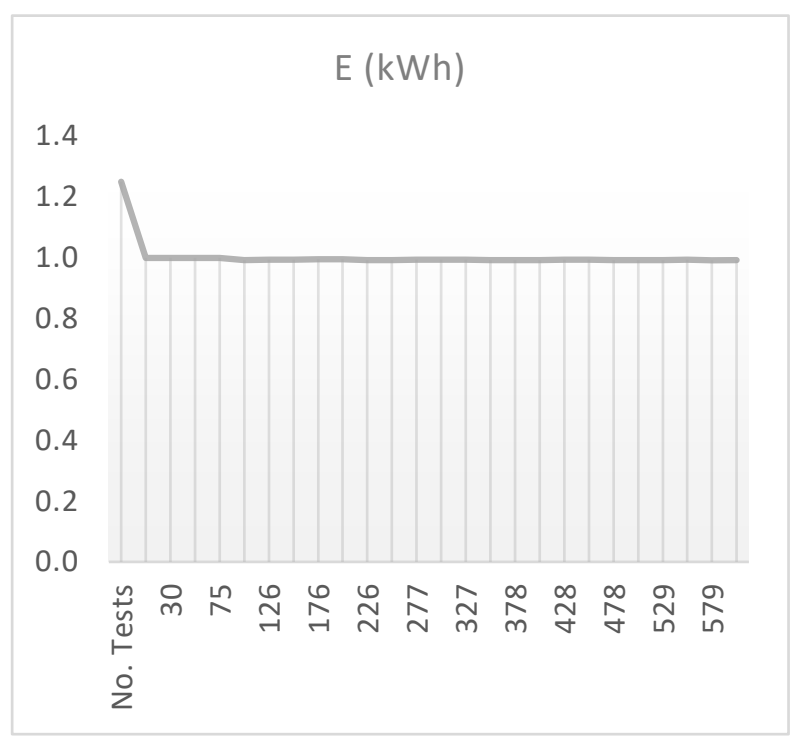

Fig. 20 The energy consumption per test versus time for one unit and using the fuzzy controller with trapezoidal $M F s$ at the best time saving of $70.6 \%$.

be used to make the crisp-controller results consistent with the experts expectations (See Section 5). The consistency can be achieved by either adjusting the limits of the adopted intervals partitioning Dtemp and Time variables, or by increasing the number of these intervals. In the first case, however, and since the results depend on the limits of the intervals, then comparatively more effort is needed to adjust and tune these limits unless a fuzzy system, where the peak values of the fuzzy sets have the major effect, is developed first and its results are used. In the second case, we will have an additional increase in the software complexity of the classical controller. Although the development and tuning of the model to accommodate experts expectations might become easier to reach than in the first case, it is still complex compared to the fuzzy model. The ease by which the fuzzy models can be designed and tuned comes from the approximate nature of the models and available specialized software tools.

Given the obtained results, the study confirms that the following objectives are met:
- Reduce the time of the refrigerant charging procedure.

- Capture the intrinsic fuzziness of the process to reduce the controller complexity.

- Capture the decision-making process of a human operator.

- Obtain results that outperform classical crisp models.

- Perform parallel testing of many refrigerators. The system includes sensors, interfaces, hardware, and application software $(G U I)$.

- Automate the processes to save labor

\subsection{Closely-Related Work}

The attempt to identify related work succeeded in matching parts of the system proposed in this investigation. The identification comprises refrigerators testing procedures and automations $[3,4,5,6,7,8,9,10,11,12,13,14$, general-purpose automatic performance testing devices that can be used in refrigerators manufacturing, and automatic refrigerant charging devices [1,2,41]. None of the identified systems attempt to fully-automate the refrigerant charging process of refrigerators.

In almost all investigations in $3,4,4,5,6,7,8,9,10,11$, 12, 13, 14. (See Section 22, the purposes include optimizing the performance of refrigeration in terms of the lowest attained temperature, power consumption, optimal charge combinations and amounts, etc. In this paper, the proposed work is analyzed according to similar performance indicators, however, it differs in the approach and application. The proposed approach aims at mimicking the behavior of a human tester, during refrigerant charging, to reach the best possible performance of refrigeration. In addition, the application of the proposed approach is on performance testing of refrigerators during the manufacturing process with automated refrigarent amount increase.

Many general-purpose end-of-line Computerized Performance Tests (CPT) devices, that can be used in refrigerators manufacturing, are available in the market. Example devices are the CPT systems by $A G R A M K O W$ [2]; the devices are equipped with advanced sensing, data acquisition, monitoring, calibration, analysis and other 
automation features. Supported automatic tests include temperature, pressure, power, air flow and humidity. Moreover, the CPT devices can be used for short-term or long-term tests of refrigerators and other similar appliances. GALILIO provides the CAPTOR $K$ data logger of field signals; the device is supported by RecData $T J$ software package. The system is interfaced to Personal Computer; it performs performance tests on cooling units and other applications. The test is carried out by collecting the temperature, current curves and other signal of the units which have been tested using the Captor $K$ device. Test may last from a minimum of 1 Second up to maximum of 99 days 1 .

A variety of automatic refrigerant charging devices are available in the market [1,2,41. Such equipment can be classified per use, such as, commercial and domestic refrigeration. Devices vary in size, refrigerant type and charge weight. Moreover, such refrigerant charging devices are used in fast-paced assembly lines with limited process variability; they aim at providing automation, process data traceability and high charging accuracy. The main functions of such devices include refrigerant charging, leak testing, etc.

\section{Conclusion}

This study has introduced a new approach mainly based on fuzzy logic and inference for making the experimental human-performed control of the refrigerant-charging process of refrigerators intelligent and automated. The automation is done while accounting for the reduction of the time needed to finish the testing and performance evaluation. The approach is motivated by the importance of the charging process in terms of achieving optimal performance and improved power consumption. It has also been motivated by the natural fuzziness that arises in the basic description of the experimental testing procedure and by how human experts per-form the charging process.

Based on the expert testing procedure (See Section 4); fuzzy and classical inference models are developed, where both show the capability of representing the intelligent human tester behavior and reflecting the viability of using inference methodology in application. From the perspective of better accommodating for approximate human reasoning in the application in hand, the fuzzy inference models are preferable. the developed models are shown to provide a suitable representation of the natural fuzziness that arises in the problem statement, and the correlation principle implementation that governs the behavior of an intelligent human expert.
Furthermore, the fuzzy model is shown (See Table 4 to require less software, development and tuning complexity compared to the classical models. The approximate reasoning aspect and the availability of fuzzy inference tools permit the fuzzy system to be designed and tuned easily to accommodate any refrigerant charging situation and experts expectation. Furthermore, inference tools permit the fuzzy models to be embedded in a computerized data acquisition, monitoring, and decision-making system containing the necessary hardware and software parts with the needed interfaces.

The provided fuzzy models are built upon reasonable assumptions regarding the trend of temperature change versus time, and the speed of data acquisition. Given a specific refrigerant charging situation, and the corresponding temperature versus time curve, then the data acquisition speed could be adjusted in a manner to make the developed fuzzy model match that situation. Indeed, the proposed models can be tuned to provide a better match. The tuning can be applied to the ranges of the used variables, the THEN parts of the rules, and the location of the supports and peak values of the fuzzy sets. This kind of tuning causes a change in the time needed to stop the testing for every value or range of values of Dtemp (See Section 7). With no doubts, the principles explained in Section 5 need always to be observed. Hence, the provided solution procedures are general and remain valid.

The obtained results confirmed that the fuzzy controller, and in its best case, can achieve a test time saving of $70.6 \%$. The saving leads to a constant energy consumption of $1 \mathrm{kWh}$ per tested unit. The best achieved test-time saving results, using the fuzzy controller, outperforms those of the crisp controllers. However, the best-case crisp and worst-case fuzzy controller perform in an almost identical manner.

Future work deals with the application of the introduced approach in various refrigerant-charging situations, and with the tuning and improvement of the provided fuzzy models to try to make them competitive with the performance of well-trained human experts. Although the minimization of the time factor is important, this minimization should not be done at the expense of detecting a temperature that significantly differs from the smallest possible one for every amount of refrigerant. If a value around the smallest possible temperature is not reached, then the calculated $C O P$ for every specific refrigerant amount would turn out to be wrong, and leads to a wrong decision on the amount of needed refrigerant. Hence, the specific charging situation, the balancing between the time reduction and accuracy of the detected temperature, as well as the experts opinion and expectation, need to be given care- 
ful considerations to obtain reliable and efficient fuzzy inference model. In addition, Future work can include investigating improvements towards fault-prone manufacturing using model-reduction techniques [42,43].

\section{Acknowledgments}

The authors would like to thank Mr. M. El-Khalili, a Senior Mechanical Engineer and Market Area Director at Eberspcher Strak GmbH, Germany, for the help and advice he provided in some paper related issues. In addition, the authors are grateful for the thorough reviews, by the editor and the anonymous reviewers, that enabled great improvements to this paper.

\section{References}

1. Galileo TP Process Equipment. Website, March 2017.

2. AGRAMKOW. Website, March 2017.

3. W.D. Adler. Refrigerator performance testing in the house-hold refrigerator industries today, March 2006.

4. LJM Kuijpers, MJP Janssen, and PJM Verboven. The influence of the refrigerant charge on the functioning of small refrigerating appliances. ASHRAE transactions, 94:813-828, 1988.

5. R Radermacher and K Kim. Domestic refrigerators: recent developments. International journal of refrigeration, 19(1):61-69, 1996.

6. I. Damaj, J. Saade, and H. Diab. Performance testing of refrigerators using fuzzy inference methodology under labview. In the 7th IEEE International Conference on Electronics, Circuits and Systems, volume 2, 17-20 December 2000.

7. Enio Pedone Bandarra Filho, Oscar Saul Hernandez Mendoza, Francisco Ernesto Moreno Garcia, and Jose Alberto Reis Parise. Energy conservation for refrigeration systems by means of hybrid fuzzy adaptive control techniques. Journal of the Brazilian Society of Mechanical Sciences and Engineering, 38(6):1753-1766, 2016.

8. Zhi Yang, Pengbin Duan, Zhonghua Li, and Xiaoguang Yang. Self-adjusting fuzzy logic controller for refrigeration systems. In Information and Automation, 2015 IEEE International Conference on, pages 2823-2827. IEEE, 2015.

9. Necati Kocyigit. Fault and sensor error diagnostic strategies for a vapor compression refrigeration system by using fuzzy inference systems and artificial neural network. International Journal of Refrigeration, 50:69-79, 2015.

10. Yu Wen You, Zhi Gang Zhang, Chun Mei Guo, and Li Lu Zhang. Optimizing approach to varying load of the refrigeration system based on fuzzy logic control. In Advanced Materials Research, volume 433, pages 6896-6901. Trans Tech Publ, 2012.

11. Hua Li and Jiyou Fei. Fuzzy control for a variable speed refrigeration system. In Measuring Technology and Mechatronics Automation (ICMTMA), 2011 Third International Conference on, volume 2, pages 272-275. IEEE, 2011.

12. Arzu Şencan Şahin, İsmail İlke Köse, and Reşat Selbaş. Comparative analysis of neural network and neuro-fuzzy system for thermodynamic properties of refrigerants. $A p$ plied Artificial Intelligence, 26(7):662-672, 2012.
13. M Mahbubur Rashid and Ashraful Islam. Design and implementation of a fuzzy logic based controller for refrigerating systems. In Computer and Communication Engineering (ICCCE), 2010 International Conference on, pages 1-5. IEEE, 2010.

14. Weiqiang Pang, Jinping Liu, and Xiongwen Xu. A strategy to optimize the charge amount of the mixed refrigerant for the joule-thomson cooler. International Journal of Refrigeration, 69:466-479, 2016.

15. LA Zedeh. Outline of a new approach to the analysis of complex systems and decision processes. IEEE Transactions on Systems, Man, and Cybernetic, 3:28-44, 1973.

16. Dimiter Driankov, Hans Hellendoorn, and Michael Reinfrank. Introduction. In An Introduction to Fuzzy Control, pages 1-36. Springer, 1996.

17. Stanislaw H Zak. Systems and control. 2003.

18. John Essick. Hands-on introduction to LabVIEW for scientists and engineers. Oxford University Press, 2013.

19. Measurement National Instruments: Test and Embedded Systems. Website, March 2017.

20. VI Dmitriyev and VE Pisarenko. Determination of optimum refrigerant charge for domestic refrigerator units. International Journal of Refrigeration, 7(3):178180, 1984.

21. Ashraf A. Zaher. Design of model-based controllers for a class of nonlinear chaotic systems using a single output feedback and state observers. Phys. Rev. E, 75:056203, May 2007.

22. Lotfi A Zadeh. Fuzzy sets. Information and control, 8(3):338-353, 1965.

23. Lotfi A Zadeh. Fuzzy logic and approximate reasoning. Synthese, 30(3):407-428, 1975.

24. George Klir and Bo Yuan. Fuzzy sets and fuzzy logic, volume 4. Prentice hall New Jersey, 1995.

25. Wen Jiang, Yu Luo, Xi-Yun Qin, and Jun Zhan. An improved method to rank generalized fuzzy numbers with different left heights and right heights. Journal of Intelligent \& Fuzzy Systems, 28(5):2343-2355, 2015.

26. Hung T Nguyen and Michio Sugeno. Fuzzy systems: modeling and control, volume 2. Springer Science \& Business Media, 2012.

27. Hans Hellendoorn and Christoph Thomas. Defuzzification in fuzzy controllers. Journal of Intelligent \& Fuzzy Systems, 1(2):109-123, 1993.

28. Naim Çăgman and Serkan Karataş. Intuitionistic fuzzy soft set theory and its decision making. Journal of Intelligent E Fuzzy Systems, 24(4):829-836, 2013.

29. Jean J Saade and Hassan B Diab. Defuzzification techniques for fuzzy controllers. IEEE Transactions on Systems, Man, and Cybernetics, Part B (Cybernetics), 30(1):223-229, 2000.

30. Y. Wei, J. Qiu, and H. R. Karimi. Reliable output feedback control of discrete-time fuzzy affine systems with actuator faults. IEEE Transactions on Circuits and Systems I: Regular Papers, 64(1):170-181, Jan 2017.

31. Y. Wei, J. Qiu, and H. R. Karimi. Fuzzy-affine-modelbased memory filter design of nonlinear systems with time-varying delay. IEEE Transactions on Fuzzy Systems, PP(99):1-1, 2017.

32. H. Zhang and J. Wang. Active steering actuator fault detection for an automatically-steered electric ground vehicle. IEEE Transactions on Vehicular Technology, 66(5):3685-3702, May 2017.

33. B. C. Lim and M. Horowitz. Error control and limit cycle elimination in event-driven piecewise linear analog functional models. IEEE Transactions on Circuits and Systems I: Regular Papers, 63(1):23-33, Jan 2016. 
Table 4 Comparison of crisp and fuzzy controllers.

\begin{tabular}{c|c|c}
\hline Indicator & $\begin{array}{c}\text { Crisp Controller } \\
\text { Equation }[7\end{array}$ & $\begin{array}{c}\text { Fuzzy Controller } \\
\text { Table } 10\end{array}$ \\
\hline Procedure required to complement the Software tool & Complex & Simple \\
\hline Development and tuning under tools such as LabVIEW & Complex & Simple \\
\hline
\end{tabular}

34. A. Nejadpak and F. Tahami. Stabilizing controller design for quasi-resonant converters described by a class of piecewise linear models. IEEE Transactions on Circuits and Systems I: Regular Papers, 61(1):312-323, Jan 2014.

35. D. Wu. Twelve considerations in choosing between gaussian and trapezoidal membership functions in interval type-2 fuzzy logic controllers. In 2012 IEEE International Conference on Fuzzy Systems, pages 1-8, June 2012 .

36. Issam Damaj and Safaa Kasbah. An analysis framework for hardware and software implementations with applications from cryptography. Computers and Electrical Engineering, 2017.

37. I. W. Damaj. A unified analysis approach for hardware and software implementations. In 2016 IEEE 59th International Midwest Symposium on Circuits and Systems (MWSCAS), pages 1-4, Oct 2016

38. David A Patterson and John L Hennessy. Computer Organization and Design RISC-V Edition: The Hardware Software Interface. Morgan kaufmann, 2017.

39. Honeywell: Single Gas Valves. Website, January 2018.

40. Pedram Samadi, Hamed Mohsenian-Rad, Vincent WS Wong, and Robert Schober. Tackling the load uncertainty challenges for energy consumption scheduling in smart grid. IEEE Transactions on Smart Grid, 4(2):1007-1016, 2013.

41. VTECH Process Equipment LLC: Refrigarent Charging Equipment. Website, January 2018.

42. Yanling Wei, Jianbin Qiu, Hamid Reza Karimi, and Mao Wang. model reduction for continuous-time markovian jump systems with incomplete statistics of mode information. International Journal of Systems Science, 45(7):1496-1507, 2014.

43. Yanling Wei, Jianbin Qiu, Hamid Reza Karimi, and Mao Wang. Filtering design for two-dimensional markovian jump systems with state-delays and deficient mode information. Information Sciences, 269:316 - 331, 2014.

\section{Appendix. List of Acronyms and Symbols}

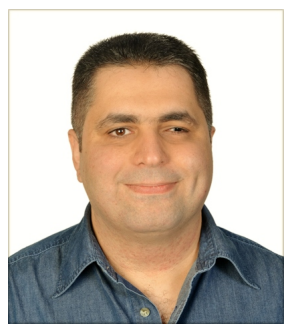

Issam W. Damaj is an Associate Professor of Computer Engineering at the Electrical and Computer Engineering (ECE) Department at American University of Kuwait (AUK) Since 2011, he has been the ABET Accreditation Lead and Institutional Representative of the ECE Department. He was the Founding Chairperson of the ECE Department and Program Lead between September 2009 and August 2016. He was awarded a Doctor of Philosophy Degree in Computer Science from London South Bank University, London, United Kingdom in 2004. He

\begin{tabular}{l|l}
\hline Acronym or Symbol & Definition \\
\hline \hline${ }^{\circ} \mathrm{C}$ & Degree Celsius \\
\hline COI & Compositional Rule of Inference \\
\hline COP & Coefficiant of Performance \\
\hline CPT & Computerized Performance Test \\
\hline DAQ & Data Acquisition \\
\hline DTemp & Temperature Change Variable \\
\hline E & Energy \\
\hline GUI & Graphical User Interface \\
\hline kWh & Kilo Watts per Hour \\
\hline max & Maximum \\
\hline$\wedge$ & Minimum Operator \\
\hline MF & Membership Function \\
\hline MISO & Multiple Input Single Output \\
\hline NI & National Instruments \\
\hline $\mathrm{P}$ & Power \\
\hline PR & Performance \\
\hline S4 & Suction Tube \\
\hline$t$ & time \\
\hline TH & Throughput \\
\hline TpD & Tests per 12-hour work Day \\
\hline TpH & Tests per Hour \\
\hline TS & Time Saving \\
\hline TT & Test Time \\
\hline Time & Observation Time Variable \\
\hline &
\end{tabular}

received a masters Degree in Computer and Communications Engineering, in 2001, from the American University of Beirut. He received a bachelors Degree in computer engineering from Beirut Arab University in 1999. His research interests include hardware/software co-design, embedded system design, automation, Internetof-things, fuzzy systems, formal methods, and engineering education. He is a Program Evaluator (PEV) with ABET Engineering Accreditation Commission (EAC), a Senior Member of the IEEE, a Professional Member of the ASEE, and a member of the Order of Engineers in Beirut. He maintains an academic website at www.idamaj.net.

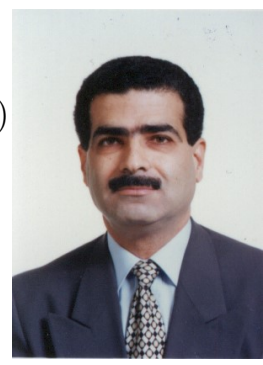

Jean J. Saade Jean J. Saade was born in Lebanon in 1954. He received the B.Sc. degree in Physics from the Lebanese University, Lebanon, in 1979 with a scholarship to pursue Ph.D. studies. He then received the M.Sc. degree in Electrical Engineering in 1982 from Boston University, MA, USA, and a Ph.D. degree, also in Electrical Engineering, from Syracuse University, NY, USA, in 1987. He taught for four years at Syracuse 
University while being a Ph.D. candidate and served for one year as a Visiting Assistant Professor at the same university after graduation. In 1988, he joined the American University of Beirut, Lebanon, and is currently a Professor at the Department of Electrical and Computer Engineering, teaching communication, signal processing and fuzzy logic courses. Dr. Saade published a good number of papers in international journals and conferences addressing the mathematical aspects and application of fuzzy sets and logic in signal detection, radar and robot navigation. He is a member of the European Society for Fuzzy Logic and Technology (EUSFLAT).

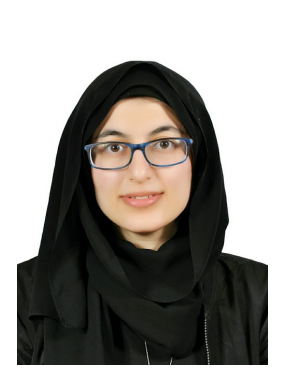

Hala R. Al-Faisal received the bachelors degree in computer engineering from the American University of Kuwait in 2016, and is currently pursuing masters degree at Kuwait University in the same field. Current research interests include artificial intelligence, and intelligent control systems.

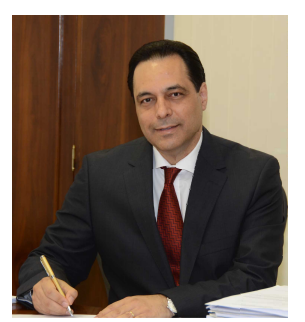

Hassan B. Diab received his B.Sc. (with Honors) in Communications, M.Sc. (with Distinction) in Systems Engineering, and Ph.D. in Computer Engineering. He joined the American University of Beirut (AUB) in 1985 and is a Professor of Electrical and Computer Engineering at the Faculty of Engineering and Architecture. He has over 140 publications in internationally refereed journals and conferences. His research interests include cryptography on high performance computer systems, modeling and simulation of parallel processing systems, embedded systems, reconfigurable computing, as well as several areas in education. He has supervised/co-supervised over 80 research projects, including 30 Masters Theses. He served as Associate Editor or member of Advisory/Editorial Board on five international journals. Professor Diab received over 20 international and regional awards. He has served as Founding Dean of the College of Engineering and Founding President during 2004-2006 at Dhofar University. Effective October 2006, Professor Diab was appointed as Vice President for Regional External Programs (REP) at AUB. He is a Founding Member of the first Arab Computer Society established in 2001 as well as the Founding Member of the IEEE Student Branch at AUB in 1997. Professor Diab is a Fellow in the IEE and the IEAust, as well as a Senior Member of the IEEE. During June 2011 February 2014, he was appointed as Minister of Education and Higher Education in the Lebanese Cabinet to take over the largest ministry in Lebanon. Since July 2013, he returned to his position as VP at AUB. 